Schreiber dieser Zeilen neun voneinander unabhängige Polbestimmungen angeführt (S. 6o) und in den Nachträgen (S. 776$)$ noch weitere vier hinzugefügt, denen möglicherweise noch andere anzureihen wären. Daselbst sind auch schon Tabellen zur Umrechnung der äquatorealen Koordinaten in galaktische erwähnt ${ }^{1}$ ).

Die vor 1870 ausgeführten Bestimmungen kommen kaum mehr in Betracht, da die frühesten guten Zeichnungen der Milchstraße von Heis und Gould stammen. Merkwürdigerweise findet sich gerade die erste genaue Bestimmung, die von Heis, soweit dem Schreiber bekannt, in der ganzen Literatur nirgends erwähnt, ausgenommen in der $>$ Himmelskunde " von Plassmann (Freiburg 1913) S. 440, wo die RA. etwas verschieden ( $\mathrm{I}^{\mathrm{h}} 4 \mathrm{I}^{\mathrm{m}} \cdot 7$ ), die Quelle aber nicht angegeben ist. Sie ist auch S. 60 des oben genannten Werkes ubersehen.

Dennoch muß bei der künftigen Wahl des galaktischen Pols dem Heisschen Werte ein bedeutendes Gewicht beigelegt

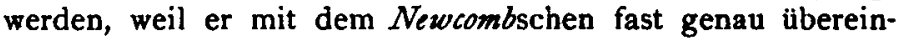
stimmt. Es handelt sich um Newcombs Berechnung, die auf beiden $Z$ weigen der Milchstraße beruht; von diesen war auch Heis ausgegangen. $\mathrm{Da}$ die Ábleitungen der beiden Werte so grundverschieden waren, die von $H$ eis rein graphisch, die von Newcomb rechnerisch, so ist die Übereinstimmung um so merkwürdiger.

Der Grund, warum die Heisschen Koordinaten bisher unbekannt geblieben sind, liegt darin, daß sie nur in der lateinischen Vorrede zum Neuen Himmelsatlas ( 1872 ) S. VIII wie nebenher angegeben sind. Daselbst heißt es: Ex omeibus observationibus meis eruitur, centrum ${ }^{2}$ ) circuli, qui dimidiat zonam viae lacteae, in puncto sphaerae situm esse, cuius ascensio recta est $190^{\circ}$ et declinatio borealis $27^{\circ}$. Newcombs Koordinaten sind bekanntlich $191^{\circ} \mathrm{I}$ und +26.8 (Contributions, Carnegie Institution, Washington I 904 S. I7).

Das Ergebnis von Heis beruht offenbar auf dem Äquinoktium seines Atlanten ( 1855 ), wie auch aus seiner Anmerkung hervorgeht, in der er die Koordinaten von $W$. Herschel erst auf 1855 überträgt, um sie dann mit den seinen zu vergleichen. Newcomb scheint absichtlich kein Äquinoktium angegeben zu haben. 'Nimmt man aber mit Innes an, er habe die galaktischen Umrisse des Atlas Coelestis novus und der Uranometria Argentina auf den gemeinsamen Äquator von 1900 zurückgeführt, so wird die Übereinstimmung mit Heis noch genauer.

Vatikan-Sternwarte, I922 Juni I 5. F. G. Hagen S.J.

1) S. 776 Zeile 9 der erwähnten Nachträge lies galaktische statt ekliptische.

ग) Heis meint hier jedenfalls das sphärische Zentrum oder den Pol.

\title{
Über die Wiederauffindung des Kometen 1916 II (Neujmin) im Jahre 1920.
}

Der kurzperiodische Komet i 9 I 6 II sollte in der zweiten Hälfte des Jahres 192 I wieder zum Perihel zurückkehren. Infolge der Abgetrenntheit der Sternwarte in Simeïs von der übrigen gelehrten Welt, die durch den Bürgerkrieg hervorgerufen war, konnte ich nicht in Erfahrung bringen, ob die bevorstehende Wiederkehr irgendwo vorausberechnet worden war. Da die mir bekannt gewordene elliptische Bahn dieses Kometen, die F. Braae im Ephemeriden-Zirkular der A. N. Nr. 506 (AN 202.317) veröffentlicht hatte, auf Beobachtungen beruhte, die sich über nur 37 Tage erstreckten, und die späteren Beobachtungen nicht befriedigend darstellte, mußten diese Bahnelemente vor der Berechnung der Ephemeride für die bevorstehende Wiederkehr verbessert werden. Die mir zugänglichen Beobachtungen umfaßten einen Zeitraum von 102 Tagen (die letzte war in Simeis am 5. Juni I 9 I 6 erhalten). Zur Verbesserung der Bahnelemente wählte ich 9 Beobachtungen (auf den Sternwarten Dearborn, Johannesburg, Mt. Hamilton, Simeïs und Williams Bay) und zog sie in drei Normalörter zusammen mit einer Zwischenzeit von 96 Tagen $z$ wischen den beiden äußersten.

Die so gefundenen neuen Elemente und die daraus ohne Berücksichtigung der Störungen abgeleitete Ephemeride für den Zeitraum 1920 Okt. 20 bis I92 I Mai 9 versuchte ich nach Frankreich zu schicken. Daß sie in der Aprilnummer I 92 I der Zeitschrift 'L'Astronomie « abgedruckt worden waren, erfuhr jch erst kürzlich aus einem Referat in Nr. 2689 der $" \mathrm{Na}$ ture«. Es scheint mir nicht überflüssig, sie hier nochmals zu wiederholen: Epoche I9I6 April 22.0 m.Z. Gr.

$$
\begin{aligned}
& M_{0}=7^{\circ} 35^{\prime} \text { 1 3.。 } \\
& \omega=1934733.0 \\
& \delta=\begin{array}{lll}
327 & 33 & 0.5
\end{array} \text { I916.0 } \\
& i=\begin{array}{llll}
\text { 10 } & 37 & \text { I } & 1.6
\end{array} \\
& \begin{aligned}
\varphi & =34^{\circ} 26^{\prime} 33^{\prime \prime 2} \\
\mu & =655^{\prime \prime} 364 \\
g a & =0.489016
\end{aligned}
\end{aligned}
$$

$$
T=\text { I9 } 6 \text { März I } 1.3239 \mathrm{~m} . \mathrm{Z} \text {. Gr. }
$$

Periode $=1977^{\mathrm{d}} \cdot 5=5^{\mathrm{a}} \cdot 4 \mathrm{I}$.

Darstellung des mittl. Ortes $\mathrm{B}-\mathrm{R}: \Delta \lambda=+0.8, \Delta \beta=-0.3$.

Wie nach der Umlaufszeit zu erwarten stand, erwies sich die Stellung des Kometen bei der zweiten Wiederkehr zum Perihel als äußerst unvorteilhaft für die Beobachtung. Der Periheldurchgang ( 10 . August I92 I) fand in der Nähe der Konjunktion des Kometen mit der Sonne statt. Die verhältnismäßig günstigsten Bedingungen boten sich Ende 1920 und Anfang 192 I dar, jedoch überstieg die Helligkeit des Kometen wegen seines großen Abstandes sowohl von der Sonne als auch von der Erde um diese Zeit nicht die Größe $15^{\mathrm{m}}$.

Da Jupiter und Saturn während des letzten Umlaufs des Kometen weit von seinem Aphel entfernt waren, konnten die Störungen, die sie ausübten, nur unbedeutend sein und ließ sich somit erwarten, daß der Komet in der Nähe des Ephemeridenorts gefunden werden könne. Infolgedessen beschloß ich den Versuch zu machen, ihn auf photographischem Wege aufzusuchen.

Zu dem Zweck machte ich am r6. November r920 eine Aufnahme nach der Metcalfschen Methode mit einer Expositionszeit von $4^{\mathrm{h}} 54^{\mathrm{m}}$. Bei der ersten Prüfung der Platte konnte ich nichts vom Kometen entdecken, bei abermaliger Untersuchung des Negativs bemerkte. ich jedoch in einer Entfernung von etwa $4^{\circ}$ vom Zentrum ein schwaches Objekt, dessen Bewegung vollständig der vorausberechneten Bewegung des Kometen entsprach. $\mathrm{DaB}$ es sich, trotz seiner außerordentlichen Lichtschwäche, um ein reelles Objekt handelte, wurde durch sein Vorkommen auf beiden Platten des Maltzoffschen Doppelastrographen béstätigt. Seine angenäherte Position war: 1920 Nov. $168^{\mathrm{h}} .7 \mathrm{~m} . \mathrm{Z} . \mathrm{Gr}$.

$\alpha$ I92I $=2^{\mathrm{h}} 0^{\mathrm{m}} \cdot 4, \delta_{\text {I92I }}=+26^{\circ} 29^{\prime}$ Gr. I $^{\mathrm{m}-16^{\mathrm{m}}}$ 
woraus eine Korrektion der Ephemeride von $-7^{\mathrm{m}} \cdot 2-3^{\circ} \mathbf{1}^{\prime}$ folgt.

Durch Variation der Durchgangszeit durch das Perihel läßt sich eine bessere Übereinstimmung zwischen der Ephemeride und dem beobachteten Ort erreichen, aber auch im günstigsten Falle, wenn man $T=$ I92 I Aug. I8.875 annimmt, beträgt der Unterschied doch noch $2^{\circ} 20^{\prime}$ im Bogen größten Kreises.

Die späte Auffindung des Kometen auf der Platte und auch die schweren Arbeitsbedingungen nahmen mir die Möglichkeit, noch weitere Aufnahmen mit ebenso langdauernder Expositionszeit zu machen. Die erwähnte Beobachtung ist daher die einzige geblieben.

Zur endgültigen Entscheidung der Frage, ob das von mir beobachtete Objekt mit dem Kometen I 9 I 6 II identisch ist, muß die wahrscheinlichste Bahn des Kometen aus den Beobachtungen des Jahres I 9 I 6 abgeleitet und eine Berechnung der Störungen bis zum Jahre i 920 ausgeführt werden; eine Arbeit, die ich bereits in Angriff genommen habe.

Pulkowo, r 922 Juni.

\section{Licht- und Farbeschwanken in kleinsten Zeiträumen. Von H. Osthoff.}

Es gibt in der Astronomie eine nicht geringe Menge von Berichten über schnell vorübergehende, flüchtige Lichterscheinungen, Lichtzuckungen, an den größten Weltkörpern. Vielfach sind diese Vorgänge als wirkliche Tatsachen angenommen worden, während man sie andrerseits bezweifelte, ohne jedoch ausreichende Erklärungen zu geben. Überblickt man die Menge von Einzelbeobachtungen dieser Art, so stellt sich sofort heraus, daß wir hier Erscheinungen mit ganz verschiedenen Ursachen vor uns haben.

Bald nachden ich begonnen hatte mit dem 4-Zöller die Farbe lichtschwächerer Sterne zu bestimmen, bemerkte ich mitunter einen Vorgang, den ich Farbenschwanken genannt habe. Er besteht darin, daß die Farbe des Sterns während des Ansehens sehr langsam ein wenig kräftiger getönt und dann wieder blasser wird. Die Periode mag durchschnittlich I-2 Minuten dauern. Der Ausschlag beträgt im stärksten Falle etwa I Stufe meiner Farbenfolge nach jeder Seite. Vorwiegend tritt das Schwanken bei den Mischfarben $6^{\circ}$ und $7^{\circ}$ ein, aber gelegentlich auch bei gelben Farbtönen. Die Dauer des Ansehens eines Sterns an sich ruft die Erscheinung nicht hervor; aber sie zeigt sich um so sicherer, je länger die Beobachtungen in die Nacht hinein ausgedehnt werden. Es gingen Jahre hin, ohne daß sie sich zeigte, während sie zu andern Zeiten häufiger auftrat.

Eine Erklärung durch Blickschwankungen trifft nicht zu. Das Farbenschwanken verläuft gleichmäBig und sehr langsam, während das unwillkürliche, geringe Schwanken des Blicks schnell zuckend vor sich geht. Ebensowenig befriedigt eine Erklärung durch das Vorüberziehen von Trübungen im Glaskörper des Auges (sog. fliegende Mücken), die sich als gewöhnliche Erscheinung in allen kurzsichtigen Augen finden. Aus meiner Beschreibung wird man ferner schon entnommen haben, daß dieses Farbenschwanken ganz und gar nichts mit dem Funkeln der Fixsterne zu tun hat. Der Unterschied zwischen dem sehr langsamen Wechsel des Farbentons und dem blitzschnellen Hin- und Herzucken der prismatischen Farben ist gar zu groß. Durch meine Versuche an künstlichen Sternen gelangte ich bald zur Überzeugung, daß das Farbenschwanken auf körperliche Anstrengung zurückzuführen ist. Die Anstrengung bei diesen Versuchen bestand in steifer, aufrechter Körperhaltung verbunden mit dem sehr langen Halten des Opernglases bei nicht gestützten Armen. Ließ ich nach jedem Anstarren des künstlichen Sterns die Arme zum Ausruhen sinken und schloß währenddessen die Augen, so ließ sich jèdesmal danach die Farbe hinreichend lange beurteilen, ohne ins Schwanken zu geraten. I9I9 Sept. 5 prüfte ich mit dem 4-Zöller lange die Färbung von $\alpha$ Cassiopeiae. Dabei rutschte das Tuch herab, mit dem ich meinen Kopf und das Okularende des Fernrohrs stets zu vẹrhüllen pflege. Um das Auge vor dem hellen Mondschein zu schützen, hielt ich die Hände links und rechts vor das Gesicht. Da entstand Farbenschwanken, sehr langsam zwischen $4^{\circ}-6^{\circ}$. Ich $20 g$ nun das Tuch wieder über and nahm eine bequeme Haltung an. Den Stern hatte ich dabei kaum aus dem Auge gelassen. Bei fortgesetztem Anschauen hörte das Farbenschwanken jetzt auf und die Farbe blieb dauernd $4^{\mathrm{c}}$. Die gegebene Erklärung trifft wohl nicht ganz zu, wenn das Schwanken eintritt, wăhrend man in bequemer Haltung vor dem Fernrohr sitzt; dann kann es sich nur um eine Anstrengung des Sehnerven allein handeln und nicht um eine allgemeine Ermüdung des ganzen Körpers.

Später bemerkte ich ähnliche Schwankungen gelegentlich bei Helligkeitsschätzungen und zwar in der Regel am selben Abend, an dem sich auch das Farbenschwanken zeigte. Daraus läßt sich auf gleiche Ursache schließen. Nur ist dieses weit leichter wahrzunehmen, weil der Farbensinn absolut ist, während beim Helligkeitsschätzen das Vergleichen mit einem andern Stern die unmittelbare Wahrnehmung benachteiligt. Auch ist die Dauer, die zur Feststellung der Helligkeit eines Veränderlichen genügt, in der Regel zu kurz, um die Lichtschwankungen hervorzurufen. Unter meinen Beobachtungen findet sich.hier und dạ eine Bemerkung, daß sich die Helligkeitsverhăltnisse unter meinen Augen wieder $z u$ ändern begannen, nachdem ich bereits zu einem sichern Urteil über die Stufenzahl gelangt war. Es kam auch gelegentlich vor, daß die Lichtgleichungen überhaupt keine Beständigkeit zeigten. In allen diesen Fällen finde ich unter den Schlußnotizen über die äußern Umstände am Beobachtungsabend die Angabe, daß ich ermüdet und abgespannt war oder $\mathrm{da} B$ mich der Gebrauch des Opernglases auf die Dauer zu sehr angestrengt hatte. Luftverhältnisse oder etwa schnell wechselndes dünnes Gewölk war nicht die Ursache. Am I 4. Oktober I92 I gegen Mitternacht wollte ich im Perseus $\epsilon$ mit $\zeta$ vergleichen. Ich stand auf dem Balkon und hielt bei hintenübergelegtem Kopfe das Opernglas mit beiden Händen. Die Haltung strengte so an, $\mathrm{daB}$ alle wiederholten Versuche fehlschlugen. Jedesmal begannen die Vergleichungen mit $\varepsilon \circ \zeta$, um schnell in ein Schwanken bis zu drei Stufen tiberzugehen. Ich unternahm einst folgenden Versuch bei dichtem Nebel, in dem fürs bloße Auge schon die Sterne 3. Größe die Grenze der Erkennbarkeit bildeten. Ich faßte mit dem Opernglase den roten $\beta$ Andromedae ins Auge, der sich nahe der Reizschwelle als rötlicher Lichtpunkt befand. Das Anstarren wurde sehr lange fort- 